Proc. Indian Acad. Sci. (Chem. Sci.), Vol. 108, No. 6, December 1996, pp. 555-573.

(C) Printed in India.

\title{
Molecular-level chiral discrimination and induction
}

\author{
KAZUHIKO SAIGO*, YUKIHIKO HASHIMOTO, \\ KAZUSHI KINBARA and ATSUSHI SUDO
}

Department of Chemistry and Biotechnology, Graduate School of Engineering, The University of Tokyo, Hongo, Bunkyo-ku, Tokyo 113, Japan

\begin{abstract}
The review describes the mechanism of chiral discrimination of racemic amines upon crystallization and the induction of chirality in organic reactions by using them as chiral auxiliaries. In order to form conglomerates, which can be resolved into the two enantiomers upon alternative seeding, both formation and packing of $2_{1}$-columns are essentially very important. On the other hand, in order to achieve high efficiency in resolution through diastereomeric salt formation, which is the most practical method, one of a pair of diastereomeric salts derived from a racemic amine and an enantiomerically pure resolving agent should at least have two 2 -columns and planar boundary surfaces in its crystal structure. On the basis of this knowledge, we developed several artificial chiral auxiliaries such as erythro2-amino-1,2-diphenylethanol, cis-2-amino-1-acenaphthenol, and cis-2-amino-3,3dimethyl-1-indanol. These were found to be very efficient chiral auxiliaries in asymmetric inductions: alkylation of chiral imines, catalytic borane-reduction, and alkylation of chiral $\mathrm{N}$-acylated oxazolidinone.
\end{abstract}

Keywords. Chiral discrimination; chiral induction; artificial chiral auxiliaries; racemic amines; diastereomeric salts.

\section{Introduction}

Continuous attention has been paid to the development of new methods for preparing enantiomerically pure compounds. Up to the present, two approaches have been mainly considered for obtaining pure enantiomers: One is the separation of the enantiomers in a racemate by applying a chiral environment. In particular, chiral discrimination upon crystallization is the most important (Jacques et al 1981a). The other is a synthetic approach, that is, the control of the stereoselectivity of a reaction by using a chiral auxiliary (see, for example, Hayashi et al 1994, Ishihara and Yamamoto 1994, Abiko et al 1995, Fuji et al 1995, Kuroki et al 1995, Maruoka et al 1995).

We have been focusing our attention on chiral discrimination of artificial chiral amines upon crystallization and on their application to organic reactions as chiral auxiliaries. In the present review, we describe our recent results on studies concerning the mechanism of chiral discrimination of racemic amines upon crystallization and the induction of chirality in organic reactions by using them as chiral auxiliaries.

\section{Chiral discrimination upon crystallization}

The methods for the optical resolution under crystalline environment are roughly classified into two categories: preferential crystallization and separation via diastereomer formation

\footnotetext{
*For correspondence
} 
(Jacques et al 1981a). We especially paid attention to the resolution of racemic amines and tried to elucidate the chiral discrimination mechanism of the amines upon crystallization, based on the crystal structures.

\subsection{Chiral discrimination upon conglomerate formation}

The simplest example of chiral discrimination upon crystallization is spontaneous resolution of racemates, i.e. conglomerate formation. The method of optical resolution applying this phenomenon is generally called preferential crystallization (Jacques $e t$ al 1981a). In the case of the resolution of amines, they are usually converted into conglomerates upon forming salts with suitable acidic derivatizing reagents (Jacques et al 1981). Although this method is one of the promising methods for optical resolution at present, the mechanism of conglomerate formation is scarcely understood. Therefore, it is almost impossible to predict and/or design a suitable derivatizing reagent from the structure of a target racemate.

In order to understand the mechanism of conglomerate formation, it is inevitable that we extract the characteristics of the crystal structures of conglomerates. On the basis of this consideration, we studied the crystal structures of the salts of chiral primary amines with achiral carboxylic acids in detail (Kinbara et al 1996a).

The crystal structures of the seven conglomerates (chart 1) revealed that a characteristic hydrogen-bond network was commonly present in all these salt crystals. Figure 1 shows an example of the crystal structure. Two ammonium cation/carboxylate anion pairs form a unit through three kinds of hydrogen bonds between the ammonium hydrogens and the carboxylate oxygens. This unit also has hydrogen bonds with translational units, forming an infinite columnar structure around a two-fold screw axis $\left(2_{1}\right.$-column, figure $\left.1 \mathrm{~b}\right)$. The alignment of the $2_{1}$-columns results in a three-dimensional crystal structure (see scheme 1). There is no significant interaction between these $2_{1}$-columns except for van der Waals interaction. Therefore, it is considered that the $22_{1}$-columns align so as to realize the closest packing where van der Waals interaction plays the most important role. Thus, it was found that all the crystal structures of these conglomerates can be regarded as supramolecular assemblies of $2_{1}$-columns.

In contrast to these conglomerates, it was found that the crystal structures of racemic-compound salts of chiral primary amines with achiral carboxylic acids could be roughly classified into two types on the basis of their hydrogen-bond networks. The first type is a crystal consisting of $2_{1}$-columns (figure 2 ), similar to those found in the
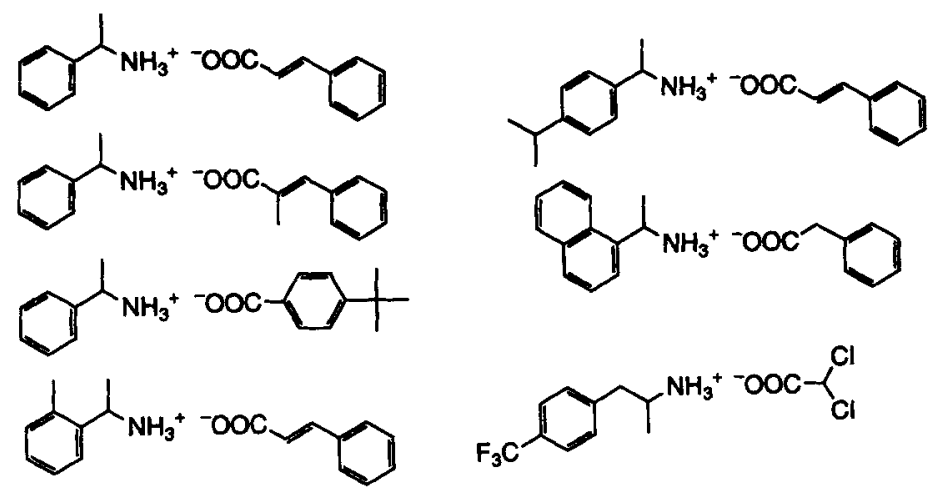

Chart 1. 
a)

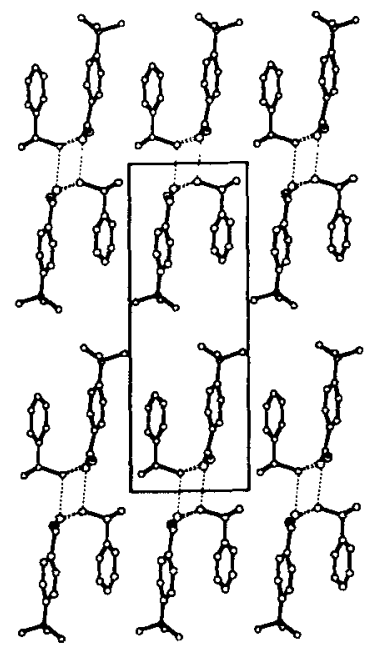

b)

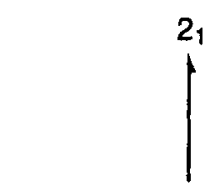

Figure 1. Crystal structure of 1-phenylethylammonium p-tert-butylbenzoate. (a) Viewed down the axis of the $2_{1}$-column ( $b$ axis). The solid and dotted lines show the unit cell and hydrogen bonds respectively. (b) $22_{1}$-Column. The dotted lines show hydrogen bonds.

conglomerate crystals mentioned above. This type of crystal structure was found for nine of the seventeen racemic compounds we studied. The $2_{1}$-column of each racemiccompound salt comprises achiral acids and chiral amines of uniform chirality (homochiral $2{ }_{1}$-column). The whole crystal is formed by the packing of two kinds of homochiral $2{ }_{1}$-columns corresponding to the two enantiomers of a chiral amine, represented as an $(R)-2_{1}$-column and an $(S)-2_{1}$-column, in the same crystal lattice (see scheme 1).

The second class of crystal found in racemic-compound salts is made of hydrogenbond columns, which have no two-fold screw axis, but rather have inversion centers (i-column) (figure 3). This type of crystal structure was found for seven of the seventeen racemic compounds. In this case, each single column comprises achiral acids and both enantiomers of a chiral amine; a single $i$-column is itself heterochiral. The whole crystal is formed by the packing of these heterochiral $i$-columns.

This classification of the crystal structures of the salts of chiral primary amines with achiral mono-carboxylic acids is summarized in scheme 1 . As is illustrated in this scheme, the formation of a $2{ }_{1}$-column is the primary requirement for conglomerate formation, and this supramolecular structure occurs in over fifty percent of the examples referred to in our study. Since a $2{ }_{1}$-column is inherently chiral, it can be said that the first chiral discrimination occurs upon formation of this chiral supramolecular structure.

Even when a homochiral $2_{1}$-column is formed preferentially, the packing mode of these $2_{1}$-columns is important. As mentioned above, it is considered that the alignment of $2_{1}$-columns in a crystal is governed only by van der Waals interaction between them. Consequently, only when the stability of $(R R)$-packing (i.e. a single crystal consists of only $(R)-2_{1}$-columns) ((SS)-packing, vice versa) overcomes the entropical advantage of 

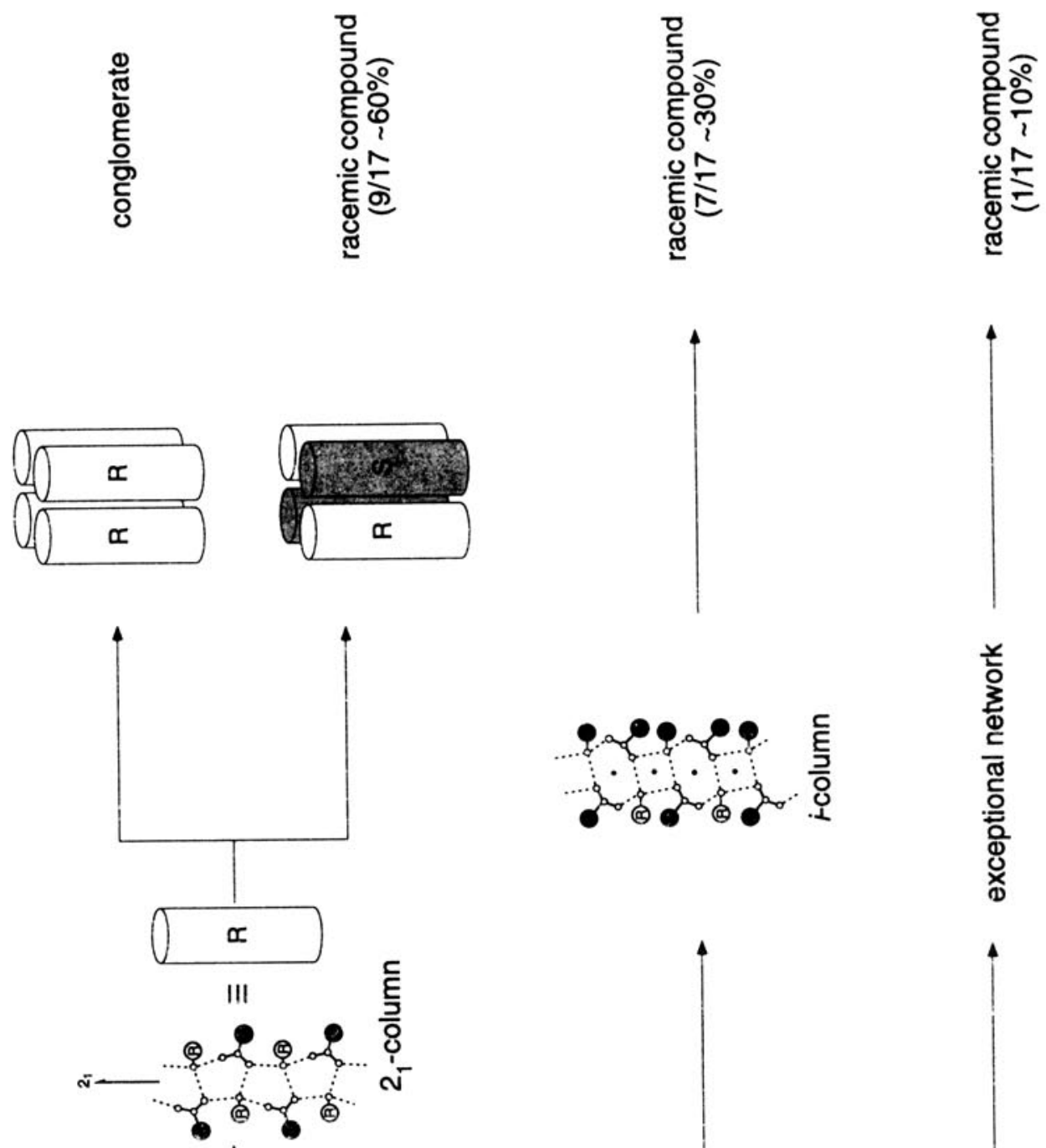

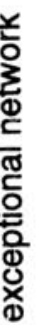

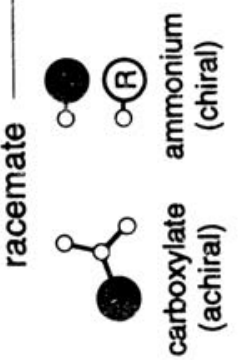




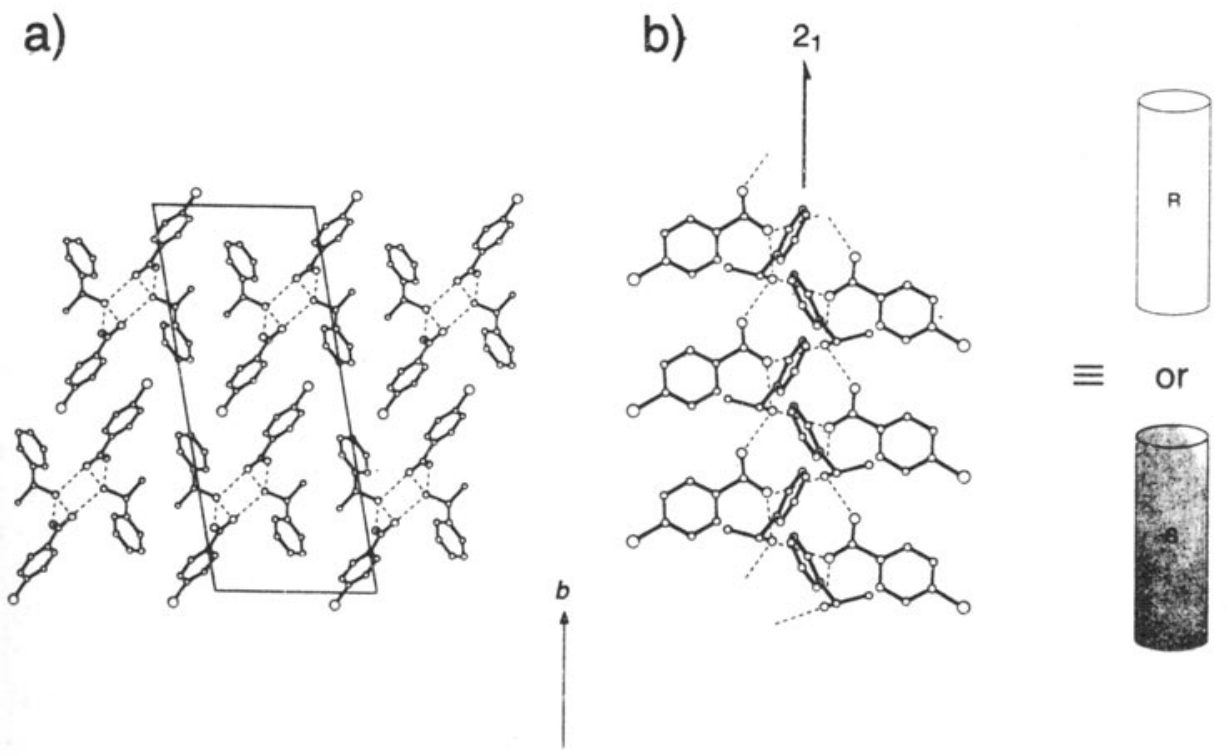

Figure 2. Crystal structure of 1-phenylethylammonium p-chlorobenzoate. (a) Viewed down the axis of the 2 -column ( $b$ axis). The solid and dotted lines show the unit cell and hydrogen bonds respectively. (b) $2_{1}$-Column. The dotted lines show hydrogen bonds.

a)

b)
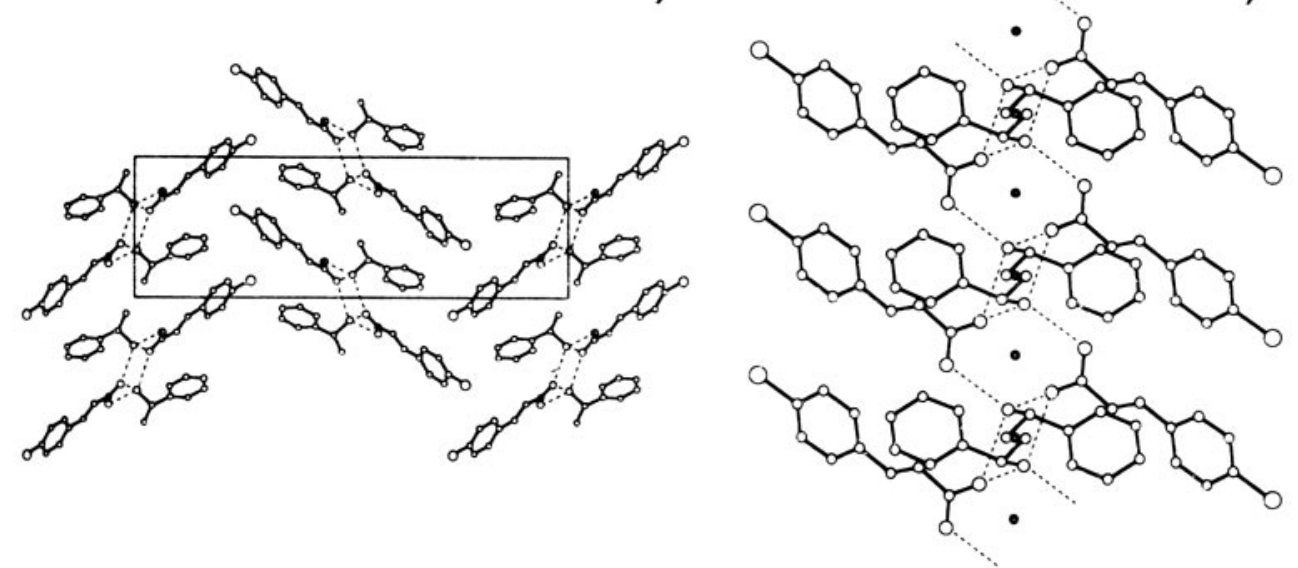

Figure 3. Crystal structure of 1-phenylethylammonium p-chlorocinnamate. (a) Viewed down the axis of the 2 -column ( $c$ axis). The solid and dotted lines show the unit cell and hydrogen bonds respectively. (b) $i$-Column. The dotted lines show hydrogen bonds.

(RS)-packing (a single crystal consists of alternate $(R)$ - and $(S)-2_{1}$-columns), will a conglomerate be formed. The realization of this situation is the secondary requirement for conglomerate formation. 
From these results, it is considered that in the case of the chiral discrimination of a primary racemic amine upon crystallization as a conglomerate, both formation and packing of 2 -columns are essentially the most important.

\subsection{Chiral discrimination upon diastereomeric salt formation}

As well as the preferential crystallization mentioned above, optical resolution of a mixture of diastereomers by recrystallization is also quite important and is the most practical method for separating enantiomers (Jacques et al 1981a). In the case of resolution of a racemic amine, an enantiomerically pure chiral acid is generally used as a resolving reagent (Newman 1981). However, it is almost impossible to predict a suitable resolving reagent for a target compound at present. We then studied the crystal structures of some diastereomeric salts and tried to design new resolving reagents based on crystal structures (Kinbara et al 1996b).

At first, we carried out the optical resolutions of racemic 1-arylethylamines (1) by $(R)$-mandelic acid $((R)-2)$ in order to understand whether there is a correlation between the structure of the amines and the efficiency of resolution by $(R)-\underline{2}$ (table 1$)$. As a result, there appeared a close relationship between the position of the substituent on the aromatic group of 1 and the resolution efficiency: $(R)-2$ could efficiently resolve 1 having a substituent at the $o$-position as well as 1-phenylethylamine (entries 2 and 3 ), whereas 1 having a substituent at the $p$-position of the phenyl group could be scarcely resolved by $(R)-2$ (entries 6 and 7); the results of $m$-substituted 1 depended on the amines (entries 4 and 5 ). These results strongly suggested that 1 , which has a similar molecular length to that of $(R)-2$, could be efficiently resolved by $(R)-2$.

We then carefully studied the crystal structures of the diastereomeric salts of 1 with $(R)-2$, which is efficiently resolved by recrystallization. As a result, in the crystals of less soluble salts, quite a characteristic hydrogen-bond network was commonly found. There are two characteristics for these hydrogen-bond networks: A $2{ }_{1}$-column is

Table 1. Resolution of 1-arylethylamines (1) by $(R)-\underline{2}$.

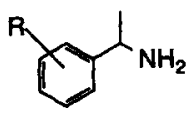

$\underline{1}$

\begin{tabular}{lllcrc}
\hline Entry & \multicolumn{1}{c}{$\mathbf{R}$} & Solvent & $\begin{array}{l}\text { Yield } \\
(\%)^{\mathbf{2}}\end{array}$ & $\begin{array}{r}\text { E.e. } \\
(\%)\end{array}$ & $\begin{array}{c}\text { Resolution } \\
\text { efficiency }\end{array}$ \\
\hline 1 & $\mathrm{H}$ & $\mathrm{H}_{2} \mathrm{O}$ & 76 & $87^{\mathrm{c}}$ & 0.66 \\
2 & $o-\mathrm{Me}$ & $\mathrm{H}_{2} \mathrm{O}$ & 71 & $100^{\mathrm{d}}$ & $0 \cdot 71$ \\
3 & $o-\mathrm{OMe}$ & $\mathrm{H}_{2} \mathrm{O}$ & 69 & $81^{\mathrm{c}}$ & $0 \cdot 56$ \\
4 & $m-\mathrm{Me}$ & $\mathrm{MeOH}$ & 94 & $12^{\mathrm{e}}$ & $0 \cdot 11$ \\
5 & $m-\mathrm{OMe}$ & $\mathrm{MeOH}$ & 70 & $89^{\mathrm{c}}$ & $0 \cdot 62$ \\
6 & $p-\mathrm{Me}$ & $\mathrm{MeOH}$ & 88 & $4^{\mathrm{d}}$ & $0 \cdot 04$ \\
7 & $p-\mathrm{OMe}$ & $\mathrm{MeOH}$ & 84 & $4^{\mathrm{e}}$ & 0.03 \\
\hline
\end{tabular}

${ }^{a}$ Yield of the crystallized diastereomeric salt based on the half amount of the racemic amine; bdefined as a product of the yield of the diastereomeric salt and the e.e. of the liberated amine; ${ }^{c}(R)$-amine was obtained as a major enantiomer; ${ }^{\mathrm{d}}(S)$-amine was obtained as a major enantiomer; ${ }^{\mathrm{e}}$ The absolute configuration of the major isomer has not been determined 
formed between ammonium groups and carboxylate groups in each case, interlinked to each other through hydrogen bonds between the hydroxyl groups of $(R)-\underline{2}$ and the carboxylate oxygens of the other $(R)-\underline{2}$, providing a rigid and stable layer structure (figure $4 a$ ), the boundary surfaces of the hydrogen-bond layer being quite planar (figure $4 \mathrm{~b}$ ). As a result, fairly close packing of the layers is realized to form a threedimensional crystal; the packing of layers is efficiently stabilized by van der Waals interaction between them. Thus, crystals of all the less-soluble salts are considered to be stable from the viewpoint of both hydrogen-bonding and van der Waals interactions.

In contrast to these less soluble diastereomeric salts, such stable crystal structures are not realized in the corresponding more soluble salts, the crystal structures of which can be roughly classified into two types. In one case, a fairly different pattern of hydrogenbond network, which seemed less stable than those of less soluble salts, was formed,
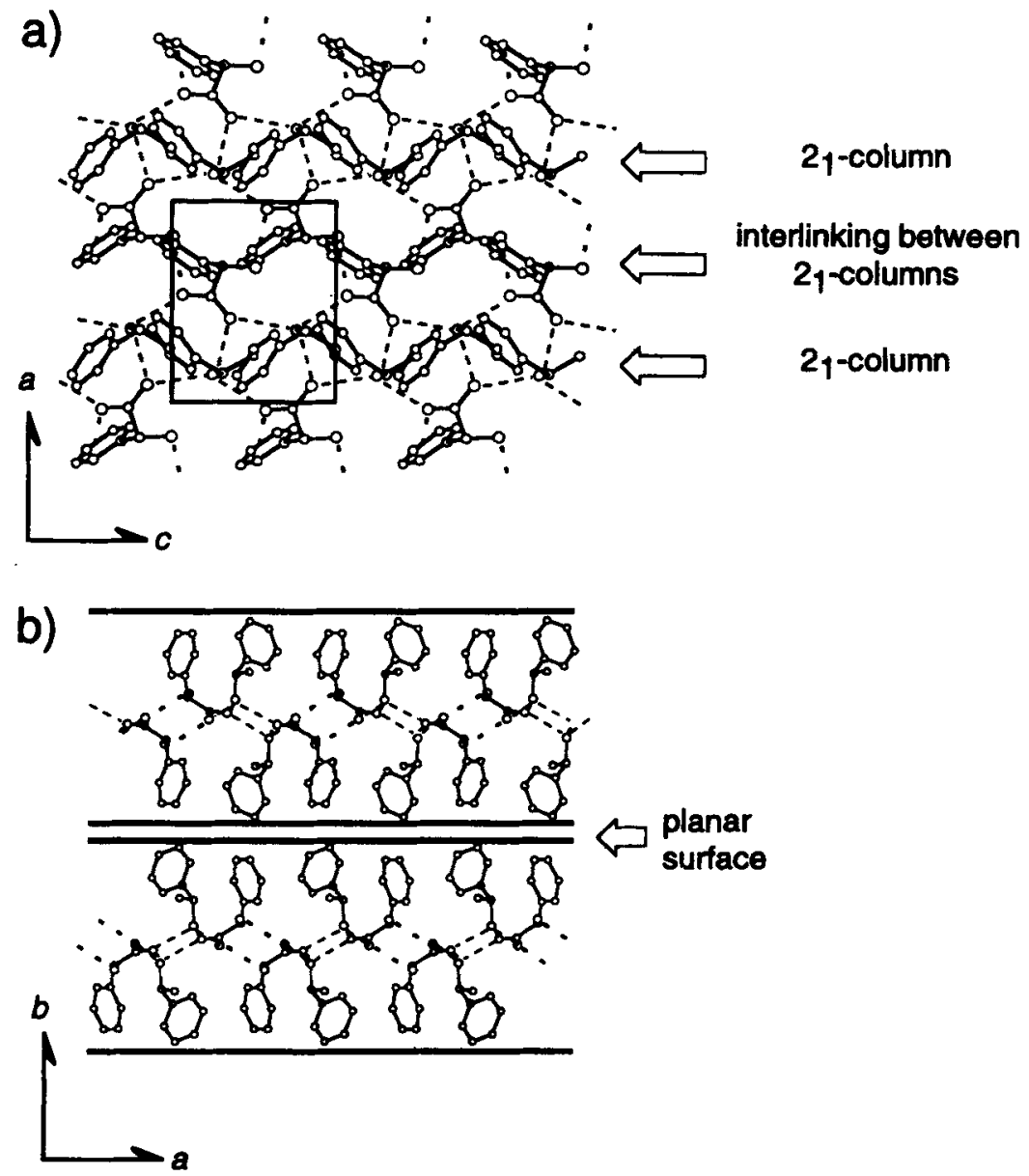

Figure 4. Crystal structure of less-soluble $(R)$-1-phenylethylammonium $(R)$-mandelate (Brianso et al 1979). (a) Viewed down the $b$ axis. The dashed lines indicate hydrogen bonds which form the columns. The dotted lines indicate the hydrogen bonds which interlink the columns. The solid lines show the unit cell. (b) Viewed down the $c$ axis. The dashed lines show the hydrogen bonds. The wavy lines show the boundary surfaces of the layer. 
although the boundary surfaces of the layer were planar and had van der Waals interactions with adjacent layers (figure 5$)^{2}$. In the other case, although a stable pattern of hydrogen-bond network consisting of $2_{1}$-columns was formed, the boundary surfaces of the layer were not planar, resulting in looser packing of the layers as compared to the less-soluble salts (figure 6).
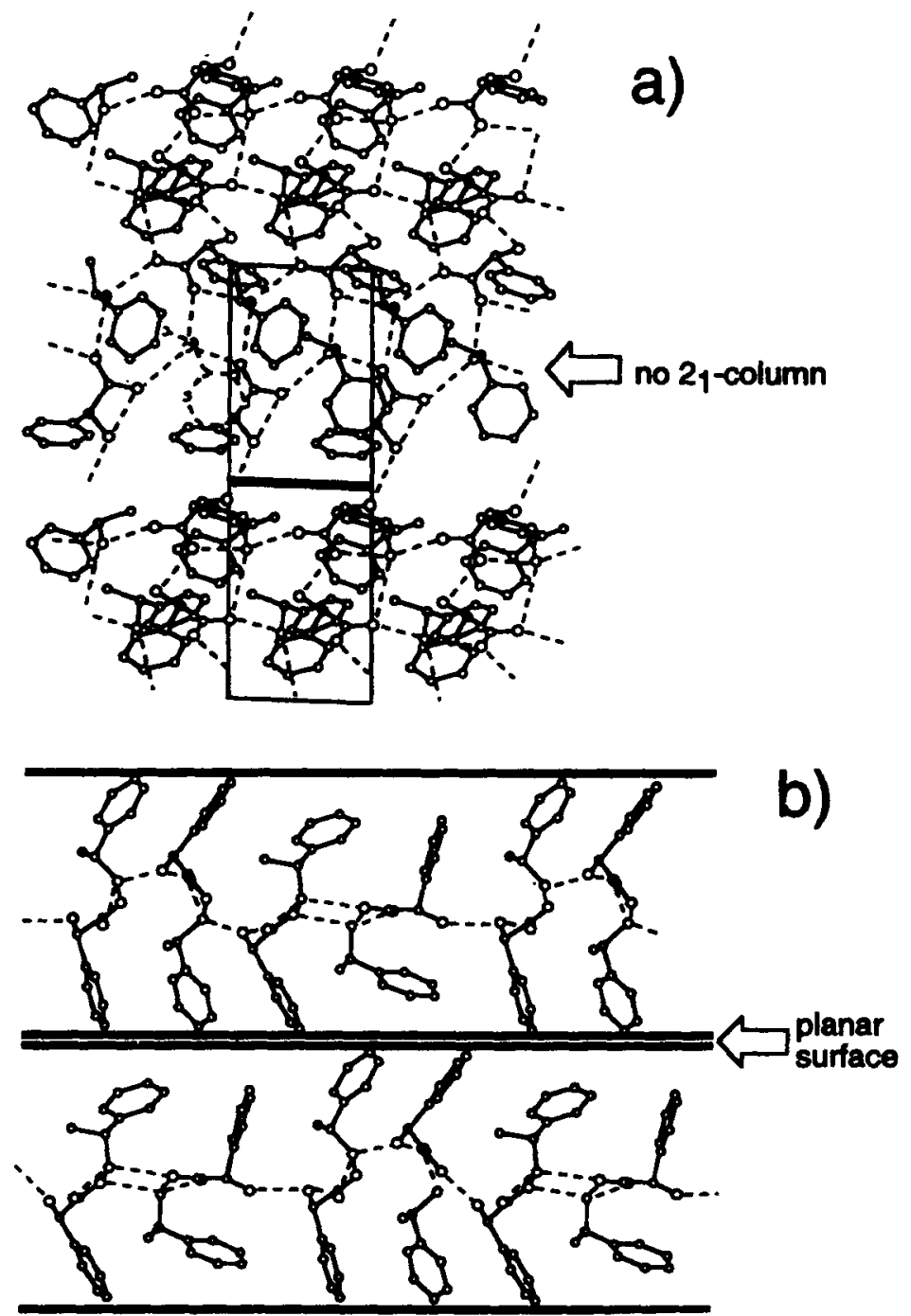

Figure 5. Crystal structure of more-soluble (S)-1-phenylethylammonium $(R)$ mandelate (Hashimoto et al 1993; de Diego et al 1994). (a) Viewed perpendicular to the hydrogen-bond layer. The solid and dashed lines show the unit cell and hydrogen bonds respectively. (b) Viewed parallel to the layer. The wavy lines show the boundary surfaces of the layer. The dashed lines show the hydrogen bonds.

\footnotetext{
${ }^{1}$ Since some of the hydrogen-acceptors are changed from the more favorable carboxylate oxygen to the less favorable hydroxyl oxygen in this more-soluble salt, this hydrogen-bond layer is considered to be less stable than those of the corresponding less-soluble salts.
} 

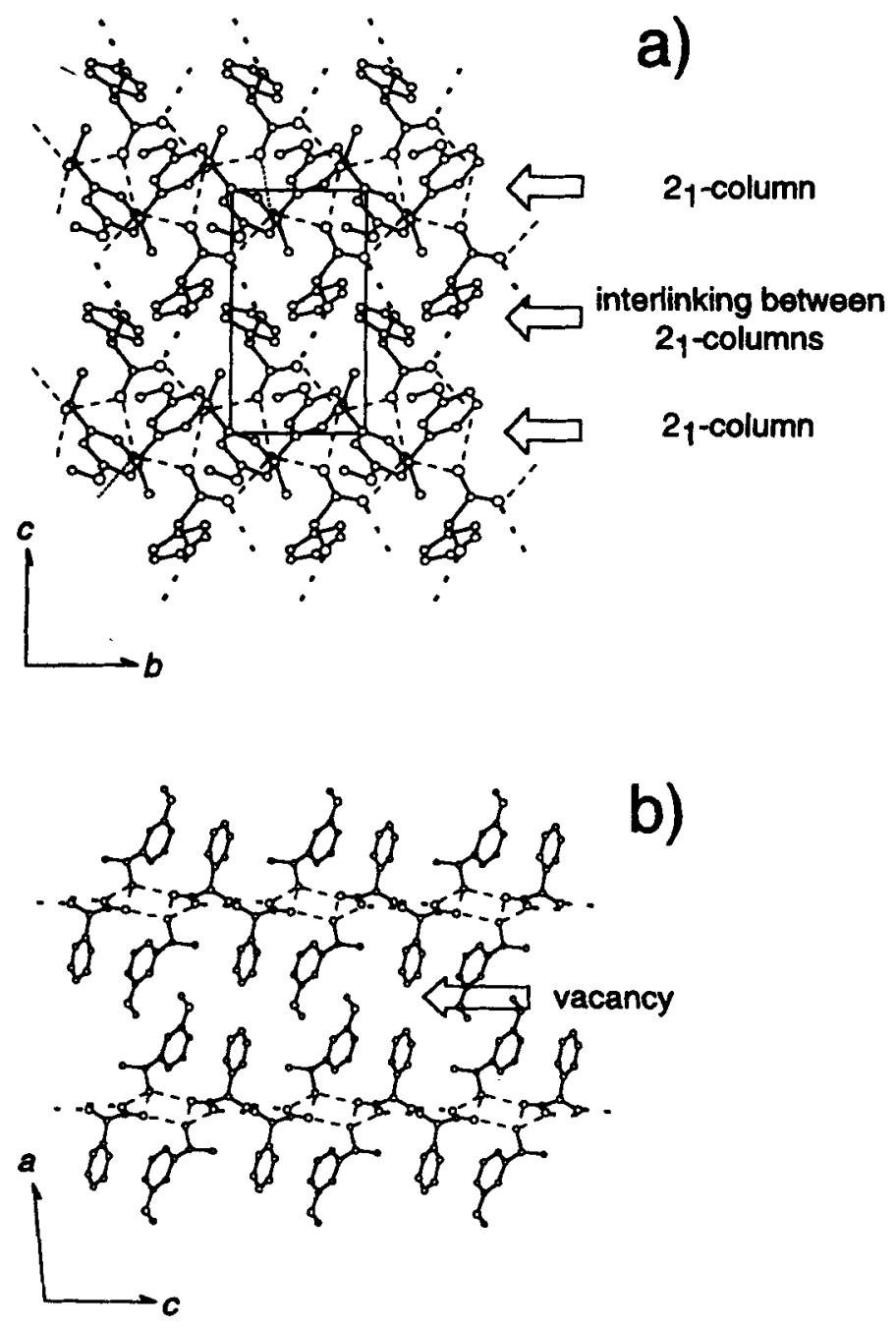

Figure 6. Crystal structure of more-soluble (S)-1-(3-methoxyphenyl)ethylammonium ( $R$ )-mandelate: (a) Viewed down the $a$ axis. The dashed lines indicate the hydrogen bonds, which form the columns. The dotted lines indicate the hydrogen bonds which interlink the columns. The solid lines show the unit cell. (b) Viewed down the $b$ axis. The dashed lines show the hydrogen bonds.

These results indicate that the less soluble salts are efficiently stabilized by two forces, hydrogen-bonding and van der Waals interactions, whereas the more soluble salts are stabilized by only one of them. Thus the less soluble salts are much more stable than the corresponding more soluble salts, resulting in the preferential precipitation of the less-soluble diastereomeric salts upon crystallization.

The crystal structures of the diastereomeric salts of $(R)-2$ with 1-( $p$-tolyl)ethylamine $(1(\mathrm{R}=p-\mathrm{Me}))$, which could not be resolved by $(R)-2$ (table 1 , entry 6$)$, were also studied. The crystal structures are similar to those of the above-mentioned more soluble salts; 
one diastereomer had a less stable pattern of hydrogen-bond network, while the packing of the layers in the other diastereomer was quite loose. From these results, it is considered that the failure of the resolution of $1(\mathrm{R}=p-\mathrm{Me})$ by $(R)-\underline{2}$ is ascribed to the projection of the $p$-substituent on the aromatic group of the amine out of the hydrogen-bond layer, which caused looser packing of the layers or another less-stable pattern of hydrogen-bond network. Namely, the effect of a substituent of 1 on the efficiency of resolution shown in table 1 can be also explained as follows: When a substituent, which enlarges the molecular length, is introduced on the phenyl group of 1 , the substituent tends to project out of the boundary surfaces of the hydrogen-bond layer, consisting of columnar hydrogen bonds (figure 7b). Subsequently, the packing of the layers becomes loose; otherwise, if such loose packing of the layers is energetically too unfavorable, another pattern of hydrogen bonds is formed to realize close packing of the layers (figure $7 \mathrm{c}$ ), although the pattern of the hydrogen bonds becomes less favorable.

We then considered that the combination of $p$-substituted mandelic acid and $\underline{1}(\mathrm{R}=p-\mathrm{Me})$ would form an ideal hydrogen-bond layer, because the $p$-substituent on the mandelic acid derivative will project out of the boundary surfaces of the layer as

a)

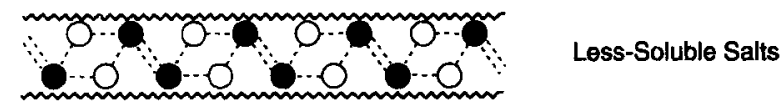

b)

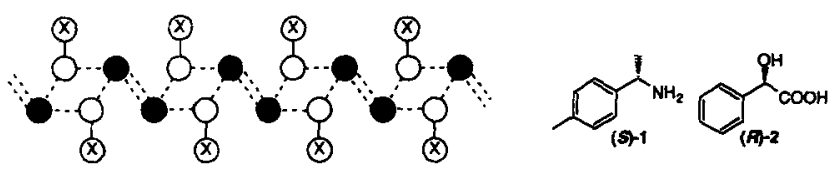

c)
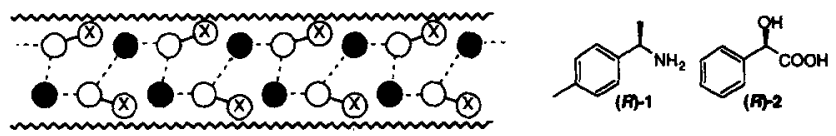

Figure 7. Schematic representations for hydrogen-bond layers in the crystals of diastereomeric salts. The white and black spheres represent 1 cation and 2 anion respectively. The dashed lines represent hydrogen bonds. (a) Hydrogen-bond layer of less-soluble salts. The wavy lines show the boundary surfaces of the layer. (b) Crystal structure of more-soluble salts (1). (c) Crystal structure of more-soluble salts (2). The wavy lines show the boundary surfaces of the layer.

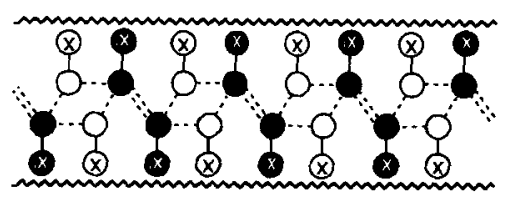

Figure 8. Schematic image of the effect of the substituent at the $p$-position of the phenyl ring of 2 . The white and black spheres represent $\underline{1}$ cation and $\underline{2}$ anion respectively. $\mathrm{X}$ and $\mathrm{X}^{\prime}$ represent the substituents at the p-position of the phenyl groups of $\underline{1}$ and $\underline{2}$, respectively. 
Table 2. Resolution of 1 -arylethylamines (1) by $p$-substituted mandelic acids $(S)$ - $\underline{3}$ and $(R)-\underline{4}$.<smiles>CC(N)c1cccc(P)c1</smiles><smiles>COc1ccc(C(O)C(=O)O)cc1</smiles>

1

2 $\underline{3}$ $\underline{4}$

\begin{tabular}{llclccc}
\hline Entry & $\mathrm{R}$ & $\begin{array}{c}\text { Resolving } \\
\text { reagent }\end{array}$ & Solvent & $\begin{array}{l}\text { Yield } \\
(\%)^{\mathrm{a}}\end{array}$ & $\begin{array}{l}\text { E.e. } \\
(\%)\end{array}$ & $\begin{array}{c}\text { Resolution } \\
\text { efficiency }\end{array}$ \\
\hline 1 & $\mathrm{H}$ & $\underline{2}$ & $\mathrm{H} \mathrm{O}$ & 76 & $87^{\mathrm{c}}$ & 0.66 \\
2 & $p-\mathrm{Me}$ & $\frac{2}{3}$ & $\mathrm{MeOH}$ & 88 & $4^{\mathrm{c}}$ & 0.04 \\
3 & $\mathrm{H}$ & $\frac{3}{3}$ & $\mathrm{MeOH}$ & 74 & $80^{\mathrm{d}}$ & 0.59 \\
4 & $p-\mathrm{Me}$ & $\underline{4}$ & $\mathrm{MeOH}$ & 85 & $63^{\mathrm{d}}$ & 0.54 \\
5 & $\mathrm{H}$ & $\underline{4}$ & $\mathrm{MeOH}$ & 70 & $89^{\mathrm{c}}$ & 0.62 \\
6 & $p-\mathrm{Me}$ & $\underline{4}$ & $\mathbf{8 5}$ & 0.61 \\
\hline
\end{tabular}

${ }^{a}$ Yield of the crystallized diastereomeric salt based on half amount of the racemic amine; ${ }^{b}$ defined as a product of the yield of the diastereomeric salt and the e.e. of the liberated amine; $(R)$-amine was obtained as a major enantiomer; ${ }^{\mathrm{d}}(S)$-amine was obtained as a major enantiomer

well as the substituent on $\underline{1}(\mathrm{R}=p$-Me) (figure 8$)$. Thus, $p$-methylmandelic acid $((S)-\underline{3})$ (Hoover et al 1974) and $p$-methoxymandelic acid ((R)-4) (Hoover et al 19'4) were selected as new resolving reagents for $\underline{1}(\mathrm{R}=p$-Me).

As a result, these acids were found to have a considerably higher resolving ability for $\underline{1}(\mathrm{R}=p-\mathrm{Me})$ than $(R)-\underline{2}$, as we had expected (table 2$)$. The ability increased in the order $(R)-\underline{2} \ll(S)-\underline{3}<(R)-4 ; p$-substituted mandelic acids were found to be fairly efficient resolving reagents for $\underline{1}$, which could scarcely be resolved by $(R)-\underline{2}$.

Thus, based on crystal structures of diastereomeric salts, we succeeded in designing resolving reagents for $p$-substituted $\underline{1}$. This is the first rational design of a suitable rẹsolving reagent.

\section{Chiral induction by artificial chiral auxiliaries}

In addition to the separation of the enantiomers of racemates, asymmetric syntheses using chiral auxiliaries are attracting much attention. So far, a number of chiral compounds have been reported as efficient chiral auxiliaries. However, since a considerable number of them are natural products and their derivatives, only one enantiomer for each compound is usually available. Moreover, it is hard to obtain them in large quantities. These disadvantages prompted us to develop new artificial chiral auxiliaries, which can be easily prepared and resolved by simple procedures.

\subsection{Erythro-2-amino-1,2-diphenylethanol}

3.1a Design, synthesis, and optical resolution: Ephedrine and its demethylated form, norephedrine, are well known to be efficient chiral auxiliaries in asymmetric syntheses. As an artificial chiral auxiliary, we at first designed erythro-2-amino-1,2diphenylethanol (5), which has a phenyl substituent in place of the methyl group in 
norephedrine, on the basis of the consideration that a phenyl group would be more effective than a methyl group in controlling the chiral environment.

Racemic amino alcohol $\underline{5}$ could be prepared on a large scale from benzoin oxime upon hydrogenation in the presence of a catalytic amount of $\mathrm{Pd} / \mathrm{C}$ (scheme 2) (Weijland et al 1951). Since this $\underline{5}$ is a racemic compound we then tried to transform $\underline{5}$ into a conglomerate upon the formation of its salt with an achiral acid. As a result, the salt of $\underline{5}$ with cinnamic acid was found to be a conglomerate, and was successfully resolved by preferential crystallization (table 3) (Saigo et al 1982).

$3.1 \mathrm{~b}$ Application to asymmetric synthesis: As an example of the application of 5 to an asymmetric synthesis, we carried out diastereofacially selective addition reactions of organometallic reagents to chiral imines $\underline{7}$, derived form $\mathrm{O}$-methylated $\underline{6}$, and various aldehydes (scheme 3) (Hashimoto et al 1995). We first examined the alkylation reaction of various chiral imines $\underline{Z}$ with alkyllithium reagents (table 4). In all cases, excellent diastereofacial selectivity was observed.

These successful results encouraged us to apply this reaction strategy to a synthetically more valuable reaction, such as an allylation reaction. As the alkylation reaction, the allylation reaction of $\underline{Z}$ was carried out using allyllithium reagents (table 5). In all cases, excellent diastereofacial selectivity was observed.

It is noteworthy that the reaction regioselectively proceeded in a 1,2-addition manner, even when an $\alpha, \beta$-unsaturated imine was used (tables 4 and 5, entry 5).

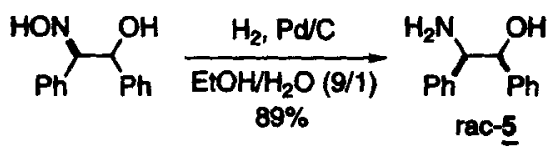

Scheme 2.

Table 3. Preferential crystallization of the salt of $\underline{5}$ with cinnamic acid.

\begin{tabular}{lccccccc}
\hline Run & $\begin{array}{c}\text { Salt } \\
\text { added }(\mathrm{g})\end{array}$ & Seed & $\begin{array}{c}\text { Cooling } \\
\text { temp }\left({ }^{\circ} \mathbf{C}\right)\end{array}$ & $\begin{array}{c}\text { Cooling } \\
\text { time/min }\end{array}$ & $\begin{array}{c}\text { Yield } \\
(\mathrm{g})\end{array}$ & $\begin{array}{c}{[\alpha]_{435}} \\
\text { degrees }\end{array}$ & $\begin{array}{c}\text { Optical } \\
\text { purity }(\%)\end{array}$ \\
\hline 1 & - & $(+)$ & 7 & 250 & 0.32 & +53.5 & 31 \\
2 & 0.40 & $(-)$ & 5 & 250 & 0.40 & -97.6 & 57 \\
3 & 0.50 & $(+)$ & 6 & 250 & 0.48 & +120.4 & 71 \\
4 & 0.70 & $(-)$ & 5 & 180 & 0.77 & -167.8 & 98 \\
5 & 0.70 & $(+)$ & 6 & 160 & 0.45 & +161.2 & 94 \\
6 & 0.80 & $(-)$ & 6 & 180 & 0.76 & -162.1 & 95 \\
7 & 0.90 & $(+)$ & 5 & 180 & 0.84 & +167.8 & 98 \\
\hline
\end{tabular}

The initial composition of the mother liquor: rac- $-5 \cdot$ cinnamic acid salt $(4 \cdot 30 \mathrm{~g})$ and rac- $\underline{5} \cdot \mathrm{lactic}$ acid salt $(6.00 \mathrm{~g})$ in $99 \%$ methanol $(110 \mathrm{ml})$. In all runs, $0.02 \mathrm{~g}$ of seed was added

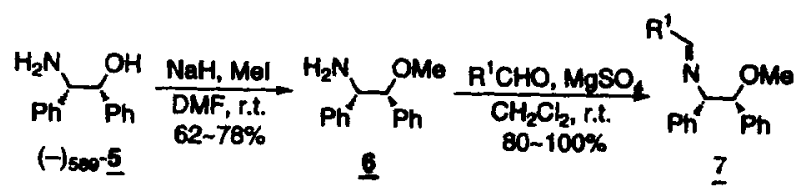

Scheme 3. 
Table 4. Alkylation reaction of chiral imines $\underline{7}$.

\begin{tabular}{|c|c|c|c|c|}
\hline Entry & $\mathbf{R}^{1}$ & $\mathbf{R}^{2}$ & Yield \% & $\underline{8}: e p i-\underline{8}^{\mathrm{c}}$ \\
\hline $1^{2}$ & $\mathrm{Ph}$ & $\mathrm{Me}$ & 89 & $99: 1$ \\
\hline $2^{b}$ & $\mathrm{Ph}$ & $n$-Bu & 64 & $99: 1$ \\
\hline 3 & $p-\mathrm{ClC}_{6} \mathrm{H}_{4}$ & $\mathrm{Me}$ & 69 & $99: 1$ \\
\hline 4 & $p-\mathrm{MeOC}_{6} \mathrm{H}_{4}$ & Me & 60 & $99: 1$ \\
\hline 5 & $\mathrm{PhCH}=\mathrm{CH}$ & Me & 43 & $99: 1$ \\
\hline 6 & $i-\operatorname{Pr}$ & $\mathrm{Ph}$ & 50 & $99: 1$ \\
\hline
\end{tabular}

"Reaction temperature: r.t.; ${ }^{\mathrm{b}}$ reaction temperature: $-78^{\circ} \mathrm{C}$ $\sim$ r.t.; "determined by GC

Table 5. Nucleophilic addition of allyllithium reagents to chiral imines 7 .
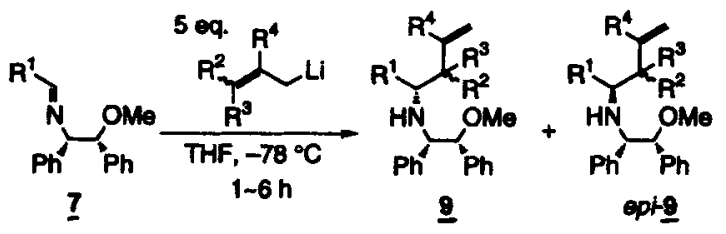

\begin{tabular}{|c|c|c|c|c|c|c|}
\hline Entry & $\mathbf{R}^{1}$ & $\mathbf{R}^{2}$ & $\mathbf{R}^{3}$ & $\mathbf{R}^{4}$ & Yield (\%) & 9:epi-9 $\underline{9}^{\mathrm{a}}$ \\
\hline 1 & $\mathbf{P h}$ & $\mathbf{H}$ & $\mathbf{H}$ & $\mathbf{H}$ & 80 & $99: 1$ \\
\hline 2 & $\mathbf{P h}$ & Me & $\mathbf{H}$ & $\mathbf{H}$ & $72^{\mathrm{b}}$ & $100^{d}: 0$ \\
\hline 3 & $\mathbf{P h}$ & $\mathbf{M e}$ & $\mathrm{Me}$ & $\mathbf{H}$ & $75^{\circ}$ & $97: 3$ \\
\hline 4 & $\mathbf{P h}$ & $\mathbf{H}$ & $\mathrm{H}$ & $\mathbf{M e}$ & 68 & 100:0 \\
\hline 5 & $\mathrm{PhCH}=\mathrm{CH}$ & $\mathbf{H}$ & $\mathbf{H}$ & $\mathbf{H}$ & 57 & $96: 4$ \\
\hline 6 & $i-\operatorname{Pr}$ & $\mathbf{H}$ & $\mathbf{H}$ & $\mathbf{H}$ & 50 & $99: 1$ \\
\hline
\end{tabular}

atermined by GC; ${ }^{b} \alpha$-adduct was formed in $18 \%$ yield; ${ }^{\mathrm{c}} \alpha$-adduct was not detected; the erythro/threo ratio of 9 was $84 / 16$; relative stereochemistry was not determined<smiles>[R]C1C=CC2C(c3ccccc3)(c3ccccc3)C(C)N1[Si]2(F)F</smiles>

Figure 9. Transition-state model for the allylation reaction of $\underline{7}$. 
Moreover it is significant that an enolizable imine bearing an $\alpha$-proton was also alkylated without any serious decrease in the chemical yield (tables 4 and 5, entry 6).

The diastereoselectivity of the reaction was explained by the transition-state model as exemplified by the reaction with allyllithium (figure 9 ). In the reaction, a rigid fused 5,6-membered ring is expected to be formed by the chelation between the Li cation and the ether oxygen of the chiral auxiliary, and the two phenyl groups of the chiral auxiliary are arranged to one side of the diastereofaces of the imine. Therefore, the direction of nucleophilic attack on the imine is strongly restricted to the opposite side of the phenyl groups to give excellent diastereofacial selectivity.

\section{2 cis-2-Amino-1-acenaphthenol}

3.2a Design, synthesis, and optical resolution: Since chiral amino alcohol $\underline{5}$ has free rotation around the single bond between the vicinal stereogenic carbons bearing the hydroxyl and amino groups, this sometimes makes any prediction of the confor-mation and the interaction with substrates in an asymmetric induction process unreliable. We therefore designed a vicinal cis-amino alcohol having a rigid skeleton, in which the orientation of the amino and hydroxyl groups is fixed by a combination of the two phenyl groups of $\underline{5}$ into a naphthalene ring. On the basis of this idea, we selected cis-2-amino-1-acenaphthenol (10) as a new chiral auxiliary.

Although the synthesis of racemic 10 has been reported, the preparation was carried out only on a small scale (Bartczak et al 1989). We then at first tried to develop a new route for the synthesis of racemic 10 . Thus, an alternative method for large-scale preparation of racemic 10 was developed via hydroxyhalogenation, followed by azidation and reduction (scheme 4) (Sudo et al 1994). This route provided a large amount of racemic 10 without complete purification in each step.

In the next stage we tried the optical resolution of racemic 10 by simple diastereomeric salt crystallization. We prepared salts of 10 with several kinds of enantiomerically pure acids, such as camphorsulfonic acid, mandelic acid, tartaric acid, malic acid, and 2-phenylpropionic acid; the diastereomeric salts were recrystallized from water, ethanol, or their mixture. Among the resolving agents examined, $(R)-2$ phenylpropionic acid was the most effective, and the examination of several conditions for recrystallization revealed that diastereomeric salt 11a was preferentially obtained in $37 \%$ yield with $98 \%$ d.e. when a 2:1 mixture of water and ethanol was used as solvent. On the other hand, recrystallization of a mixture of the diastereomeric salts from ethyl acetate predominantly gave the opposite diastereomer $\underline{11 \mathrm{~b}}$. Then, upon combining the

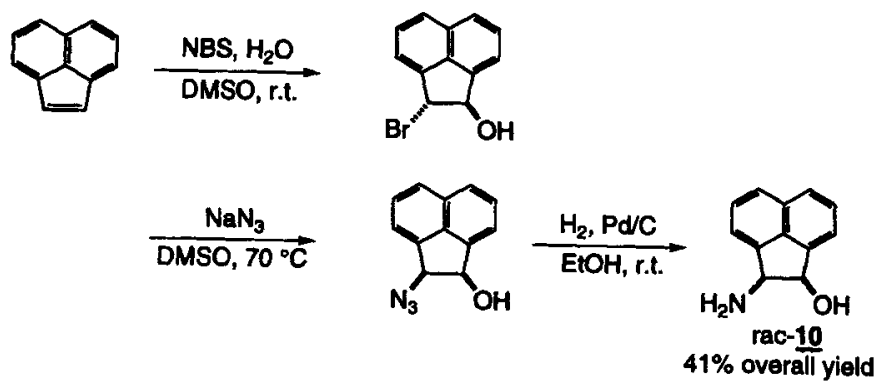

Scheme 4. 
two recrystallization processes using two different kinds of solvents, both enantiomers of $\underline{10}$ became available very efficiently (scheme 5$)^{2}$.

3.2b Application to asymmetric synthesis:. With enantiomerically pure 10 in hand, we then applied this novel chiral auxiliary to an asymmetric synthesis. Since 10 has a structural characteristic in which the orientation of the amino and hydroxyl groups is fixed due to the fused ring system of acenaphthene, we considered that 10 would be suitable to construct a rigid transition state for a catalytic reduction. On the basis of this consideration, we applied this amino alcohol for the synthesis of a chiral oxazaborolidine, which could be used in a catalytic enantioselective reduction of ketones by borane (Sudo et al 1995).

Enantiomerically pure oxazaborolidine, $\underline{12 \mathrm{a}}$ and $\underline{12 b}$, were easily prepared by the reaction of $\mathrm{BH}_{3}$.THF complex with (-)-10 and (+)-10, respectively (scheme 6 ), and used without either isolation or purification. The reduction of acetophenone was carried out using a catalytic amount of $12 \mathrm{a}(10 \mathrm{~mol} \%)$, prepared in situ, and 2 equimolar amounts of $\mathrm{BH}_{3}$.THF complex to give $(R)-1$-phenylethanol of $95 \%$ e.e. In a similar

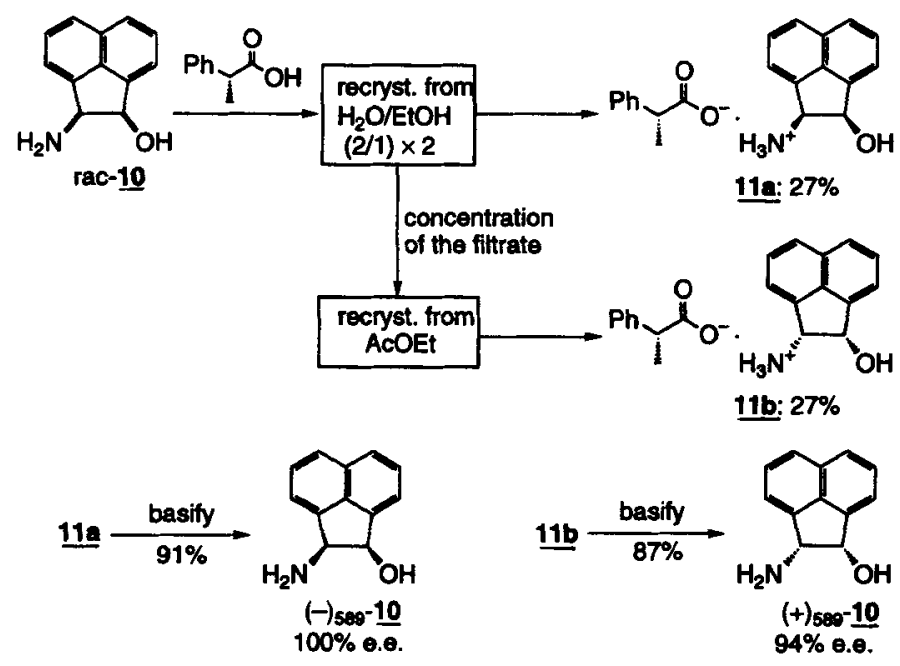

Scheme 5.
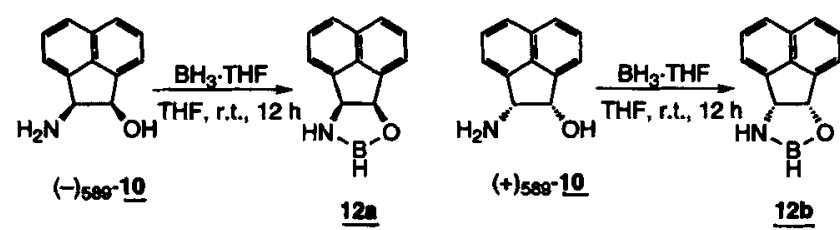

Scheme 6.

\footnotetext{
${ }^{2}$ The absolute configuration of $(-)_{589}-10$ was determined by a single-crystal X-ray structural analysis of the diastereomeric ester of $\bar{N}$ - $t$-butoxycarbonylated $(-)_{589}-10$ with $(S)$-2-phenylpropionic acid. On the basis of the absolute configuration of (S)-2-phenylpropionic acid, the absolute configuration of $(-)_{589}-10$ was determined to be $1 R, 2 S$ (Hashimoto et al 1995).
} 
Table 6. Catalytic enantioselective reduction of various ketones.

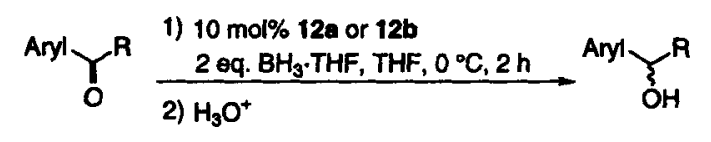

\begin{tabular}{lcccc}
\hline Entry & Cat. $^{\text {a }}$ & \multicolumn{1}{c}{ Ketone } & Yield (\%) & $\%$ e.e. (Config.) \\
\hline 1 & $\underline{12 \mathrm{a}}$ & $\mathrm{PhCOCH}_{3}$ & 89 & $95^{\mathrm{c}}(R)$ \\
2 & $\underline{12 \mathrm{~b}}$ & $\mathrm{PhCOCH}_{3}$ & 65 & $94^{\mathrm{c}}(S)$ \\
3 & $\underline{12 \mathrm{~b}}$ & $\mathrm{PhCOCH}_{2} \mathrm{CH}_{3}$ & 70 & $80^{\mathrm{d}}(S)$ \\
4 & $\underline{12 \mathrm{a}}$ & & 87 & $92^{\mathrm{d}}(R)$ \\
& & $12 \mathrm{a}$ & 81 & $91^{\mathrm{d}}(R)$ \\
\hline
\end{tabular}

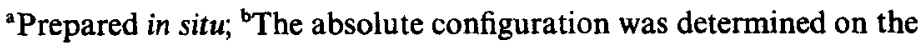
basis of the sign of the optical rotation; ${ }^{c}$ determined by a chiral HPLC analysis (Daicel Chiralcel OJ); determined by a $270 \mathrm{MHz}{ }^{1} \mathrm{H}-\mathrm{NMR}$ analysis of the MTPA ester of the product
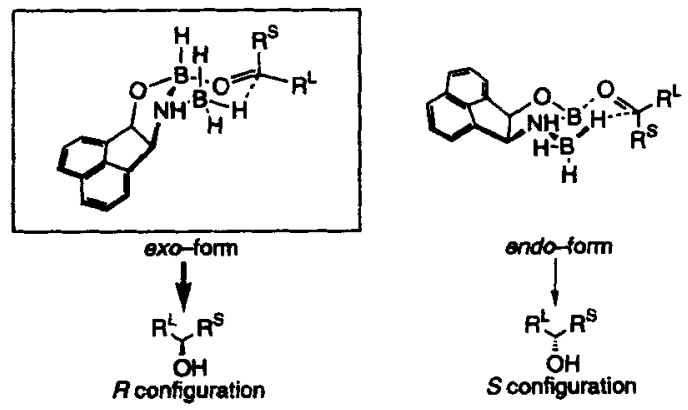

Figure 10. Transition-state models for the enantioselective reduction catalyzed by 12.

manner, the reduction of several kinds of prochiral ketones gave the corresponding alcohols in good-to-excellent selectivity (table 6). The absolute configurations of the products were in good agreement with the expectation based on a proposed transitionstate model for reduction with a chiral oxazaborolidine $/ \mathrm{BH}_{3}$ complex (figure 10).

\section{3 cis-2-Amino-3,3-dimethyl-1-indanol}

3.3a Design, synthesis, and optical resolution: Amino alcohol 10 was found to be a very efficient artificial chiral auxiliary, as expected, in a catalytic asymmetric reduction of ketones with borane. However, when 10 was applied in the asymmetric [2,3]-Wittig sigmatropic rearrangement, 10 did not work well as a chiral auxiliary (diastereoselectivity: 82/18-59/41) (Sudo et al 1994). This unsatisfactory result was considered to arise from insufficient control of the chiral environment around the chiral center adjacent to the amino group in the 5-membered ring of oxazolines, derived from 10 . On the basis of this consideration, we designed a new artificial chiral amino alcohol, cis-2-amino-3,3-dimethyl1-indanol (13) so as to have the following structural characteristics: (i) The orientation of 
the amino and hydroxyl groups is fixed. (ii) The bulkiness of the two methyl substituents strongly influences the chiral environment near the chiral center adjacent to the nitrogen atom.

Racemic 13 was easily synthesized from 3,3-dimethyl-1-indanone, as shown in scheme 7. We examined the optical resolution of racemic 13 via diastereomeric salts with several kinds of chiral acids, such as camphorsulfonic acid, tartaric acid, and mandelic acid. Among the resolving agents examined, (S)-mandelic acid was the most effective: twice recrystallization gave diastereomerically pure 14 in $35 \%$ overall yield (scheme 8$)^{3}$ (Sudo and Saigo 1995).

3.3b Application to asymmetric synthesis: In the next stage we tried to apply this new chiral amino alcohol as a precursor of a chiral auxiliary for asymmetric synthesis. First, chiral oxazolidinone 16 was prepared and used in the alkylation reaction of a carboxylic acid derivative.

Substrate 16 was obtained by $\mathrm{N}$-acylation of $\underline{15}$ without any problem (scheme 9). The lithium enolate of 16 formed upon treating it with LDA, was easily alkylated with

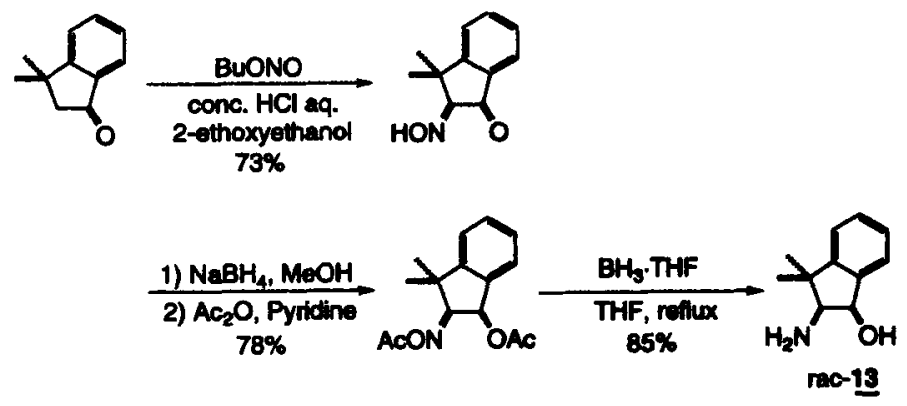

Scheme 7.

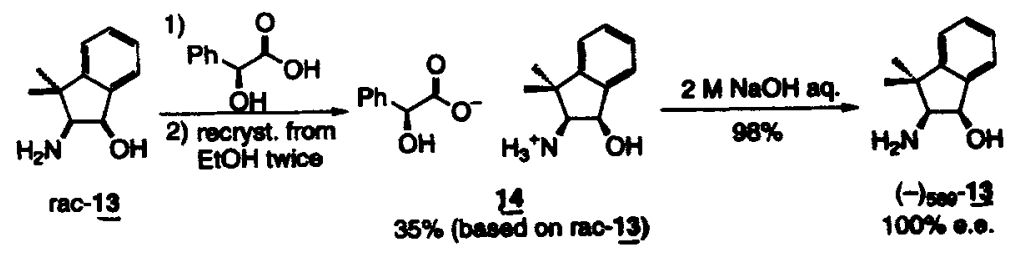

Scheme 8.

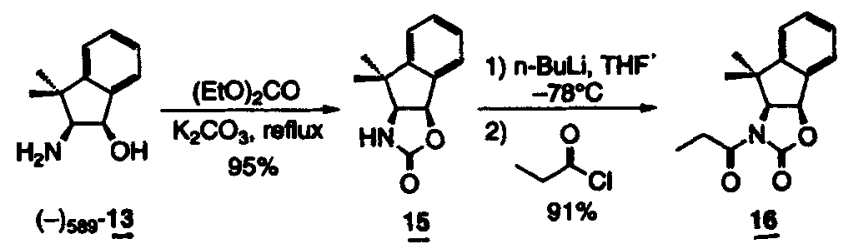

Scheme 9.

\footnotetext{
${ }^{3}$ The absolute configuration of amino alcohol $(-)_{589}-13$ was determined by a single-crystal $\mathrm{X}$-ray structural analysis of salt 14 . On the basis of the absolute configuration of $(S)$-mandelic acid, the absolute configuration of $(-)_{589}-13$ was determined to be $1 R, 2 S$ (Sudo et al 1995).
} 
Table 7. Diastereoselective alkylation of lithium enolate of $\underline{16}$.

\begin{tabular}{|c|c|c|c|}
\hline Entry & $\mathbf{R X}$ & Yield $(\%)$ & Ratio (17:epi-17) \\
\hline 1 & EtI & 57 & $>99: 1$ \\
\hline 2 & AllylBr & 95 & $>99: 1$ \\
\hline 3 & $\mathrm{BnBr}$ & 100 & $>99: 1$ \\
\hline
\end{tabular}

${ }^{2}$ Determined by HPLC analysis

various alkyl halides. The results are given in table 7 . While no optimization was performed in order to improve the yields and selectivities, excellent diastereoselectivities were observed in all cases. These results are superior compared to those obtained by using oxazolidinones derived from natural compounds (Evans et al 1982).

\section{Closing remarks}

On the basis of the studies concerning the crystal structures of the conglomerate salts and diastereomeric salts of chiral primary amines with carboxylic acids, we demonstrated the significant effect of the supramolecular hydrogen-bond networks formed in these crystals, on the efficiency of chiral discrimination upon crystallization. These results showed that the design of a suitable derivatizing or resolving reagent would become possible based on crystal engineering.

On the other hand, on the basis of the structure of norephedrine, we successively designed artificial chiral auxiliaries, erythro-2-amino-1,2-diphenylethanol (5), cis-2amino-1-acenaphthenol (10), and cis-2-amino-3,3-dimethyl-1-indanol (13). Their racemic modifications could be prepared through simple reaction routes and optically resolved by preferential crystallization or diastereomeric crystallization. Highly efficient asymmetric syntheses were realized by using these artificial chiral auxiliaries.

Our results suggest great possibility of being able to develop more attractive methods for preparing enantiomerically pure materials. For further advance, more effective derivatizing and resolving reagents, and artificial chiral auxiliaries should be developed.

\section{Acknowledgements}

This work was supported by Grant-in-Aid for Scientific Research from the Ministry of Education, Science, Sports and Culture of Japan and by Kumagai Foundation for Science and Technology, Japan. 


\section{References}

Abiko A, Moriya O, Filla S A and Masamune S 1995 Angew. Chem., Int. Ed. Engl. 34793

Bartczak A W, Sangaiah R, Kelman D J, Toney G E, Deterding L J, Charles J, Marbury G D and Gold A 1989 Tetrahedron Lett. 301109

Brianso P M-C, Leclercq M and Jacques J 1979 Acta Crystallogr. B35 2751

de Diego H L 1994 Acta Chem. Scand. 48306

Evans D A, Ennis M D and Mathre D J 1982 J. Am. Chem. Soc. 1041737

Fuji K, Kawabata T, Kuroda A and Taga T 1995 J. Org. Chem. 601914

Hashimoto K, Sumida Y, Terada S and Okamura K 1993 J. Mass Spectrom. Soc. Jpn. 4187

Hashimoto Y, Takaoki K, Sudo A, Ogasawara T and Saigo K 1995 Chem. Lett. 235

Hayashi T, Ohno A, Lu S, Matsumoto Y, Fukuyo E and Yanagi K 1994 J. Am. Chem. Soc. 116 4221

Hoover J R E, Dunn G L, Jakas D R, Lam L L, Taggart J J, Guarini J R and Phillips L 1974 J. Med. Chem. 1734

Ishihara K and Yamamoto H 1994 J. Am. Chem. Soc. 1161561

Jacques J, Collet A and Wilen S H 1981a Enantiomers, racemates and resolutions (New York: John Wiley and Sons)

Jacques J, Leclercq M and Brienne M-J 1981b Tetrahedron 371727

Kinbara K, Hashimoto Y, Sukegawa M, Nohira H and Saigo K 1996a J. Am. Chem. Soc. 118 3441

Kinbara K, Sakai K, Hashimoto Y, Nohira H and Saigo K 1996b Tetrahedron: Asym. 71539

Kinbara K, Sakai K, Hashimoto Y, Nohira H and Saigo K 1996c J. Chem. Soc., Perkin Trans. 2 (in press)

Kuroki T, Hamada T and Katsuki T 1995 Chem. Lett. 339

Maruoka K, Saito S and Yamamoto H 1995 J. Am. Chem. Soc. 1171165

Newman P 1981 Optical resolution procedures for chemical compounds (New York: Optical Resolution Information Center) vol. 1

Saigo K, Ogawa S, Kikuchi S, Kasahara A and Nohira H 1982 Bull. Chem. Soc. Jpn. 551568

Sudo A and Saigo K 1995 Tetrahedron: Asym. 62153

Sudo A, Hashimoto Y, Kimoto H, Hayashi K and Saigo K 1994 Tetrahedron: Asym. 51333

Sudo A, Matsumoto M, Hashimoto Y and Saigo K 1995 Tetrahedron: Asym. 61853

Weijland J, Pfister K, 3rd, Swanezy E F, Robinson C A and Tishler M 1951 J. Am. Chem. Soc. 73 1216 